\title{
ON MORITA THEORY FOR SELF-DUAL MODULES
}

\author{
WOLFGANG WILLEMS AND ALEXANDER ZIMMERMANN
}

\begin{abstract}
Let $G$ be a finite group and let $k$ be a field of characteristic $p$. It is known that a $k G$-module $V$ carries a non-degenerate $G$-invariant bilinear form $b$ if and only if $V$ is self-dual. We show that whenever a Morita bimodule $M$ which induces an equivalence between two blocks $B(k G)$ and $B(k H)$ of group algebras $k G$ and $k H$ is self-dual then the correspondence preserves self-duality. Even more, if the bilinear form on $M$ is symmetric then for $p$ odd the correspondence preserves the geometric type of simple modules. In characteristic 2 this holds also true for projective modules.
\end{abstract}

\section{INTRODUCTION}

Let $k$ be a field and let $G$ be a finite group. It is well-known that a $k G$-module $V$, simply called a $G$-module, carries a non-degenerate $G$-invariant $k$-bilinear form if and only if $V$ is isomorphic to its dual $V^{*}=\operatorname{Hom}_{k}(V, k)$. Up to an automorphism of $V$, a simple self-dual $G$-module $V \cong V^{*}$ carries exactly one $G$-invariant bilinear form different from the zero. In case $k$ is large enough, for instance $k$ algebraically closed, this means that such a form, if it exists, is unique up to a scalar. If in addition the characteristic $p$ of $k$ is odd this form is symmetric or antisymmetric. The case $p=2$ turns out to be more subtle. Note that in this case an antisymmetric form is symmetric. By Fong's Lemma (4], Chap. VII, Theorem 8.13), a simple self-dual $G$-module $V$ different from the trivial module always carries a non-degenerate $G$-invariant symplectic form. However, it may happen that $V$ even has a non-degenerate $G$-invariant quadratic form. This holds true, for instance, if $G$ is solvable but not in general. What really happens is not an easy task to decide; it is a question in cohomology (see [11]), at least for arbitrary groups. To be brief throughout the paper we call a $G$-module of symmetric, antisymmetric, symplectic, resp. quadratic type if $V$ carries a non-degenerate $G$-invariant form of symmetric, antisymmetric, symplectic, resp. quadratic type. The reader not familiar with duality theory of modules may refer Chapter VII of [4].

In representation theory of finite groups $G$ we are often interested in the category of modules belonging to a $p$-block $B_{G}$ of $k G$ where the underlying field $k$ is of characteristic $p$. Instead of investigating $B_{G}$ we may study a Morita equivalent $p$-block $B_{H}$ for some other group $H$. Recall that one calls two blocks Morita equivalent if their module categories are equivalent as exact categories. Morita theory shows that the module categories of the two blocks are equivalent if and only if there is an $H \times G$-bimodule $M={ }_{H} M_{G}$ where $M$ is a progenerator for $B_{H}$ and for $B_{G}$. Moreover the functor

$$
V \mapsto M \otimes_{k G} V
$$

defines an equivalence from the module category of $B_{G}$ onto that of $B_{H}$. The correspondence preserves all functorial properties of module categories, in particular being simple, indecomposable, projective, Loewy structure and the like. For more details the reader is referred to Chapter 4 in [10].

Date: May 13, 2007; revised March 25, 2008.

The first author is supported by a German-French grant "PROCOPE" of the DAAD, the second by a French-German grant "partenariat Hubert Curien PROCOPE dossier 14765WB". Both authors gratefully acknowledge financial support. 
In this note we study the behaviour of self-duality including the type of forms under Morita equivalence. Given a group $\Gamma$ recall that a $\Gamma$-module $T$ carries a non degenerate $\Gamma$-invariant bilinear form if and only if $T$ is self-dual. A first step to our main result, useful in its own right, is the following.

Theorem A. Let $B_{G}$ and $B_{H}$ be blocks for $G$ and $H$ respectively. Suppose that the $H \times G$ bimodule $M={ }_{H} M_{G}$ defines a Morita equivalence between $B_{G}$ and $B_{H}$ and suppose that $M$ is self-dual. Then a $B_{G}$-left module $V$ is self-dual if and only if $M \otimes_{k G} V$ is a self-dual $B_{H}$-left module.

We present now our main result for algebraically closed fields $k$ of odd characteristic. Given a group $\Gamma$ we call a self-dual $k \Gamma$-module $T$ of symmetric type if $T$ carries a non degenerate $\Gamma$-invariant symmetric bilinear form. If $T$ is simple and self-dual the $\Gamma$-invariant form is unique up to a scalar and the form is then either symmetric or antisymmetric. We call this the type of the simple module $T$.

Theorem B. Let $k$ be an algebraically closed field of odd characteristic. Let $M$ be an $H \times G$ bimodule inducing a Morita equivalence between a $k G$-block $B_{G}$ and a $k H$-block $B_{H}$. If $M$ is of symmetric type then the equivalence preserves the type of simple self-dual modules.

The main result for fields of characteristic 2 is weaker. We call a $k \Gamma$-module $T$ of quadratic type if $T$ admits a $\Gamma$-invariant non degenerate quadratic form.

Theorem C. Let $k$ be an algebraically closed field of characteristic 2. Let $M$ be an $H \times G$ bimodule inducing a Morita equivalence between a $k G$-block $B_{G}$ and a $k H$-block $B_{H}$. If $M$ is of symmetric type then the equivalence preserves the property "being of quadratic type" on projective modules.

Theorem A, Theorem B and Theorem C give invariants under Morita equivalence in case the Morita bimodule which induces the equivalence is self-dual for Theorem A, or even more of symmetric type for Theorem B and Theorem C. Moreover, we show that this is actually a property of the module categories, rather than of the particular bimodule. Indeed Remark 3.5 shows that if $M$ is a self-dual Morita bimodule inducing an equivalence between two blocks of group algebras, then any Morita bimodule between these blocks is self-dual. If the Morita bimodule is moreover of symmetric type, then every Morita bimodule between these blocks is self-dual.

Conditions on the Morita equivalence are actually necessary. The assertions of the theorems are false if we do not require suitable conditions on the Morita module. If the two blocks $B_{G}$ and $B_{H}$ of the group $G$ and the group $H$ are Morita equivalent, it happens that every simple module over $B_{G}$ is self-dual whereas no simple module over $B_{H}$ is self-dual. In particular, no self-dual Morita bimodule exists in this case. An example is given by a non principal 7-block of the triple cover of the McLaughlin group and the principal 7-block of the Mathieu group $M_{23}$. For details see Example 2.3 below. Even worse, two Morita equivalent blocks $B_{G}$ and $B_{H}$ may admit a self-dual Morita bimodule, but the types of the forms on the simple modules of $B_{G}$ and of $B_{H}$ are different. Hence it may happen that no Morita bimodule can be of symmetric type. An example is given by a non principal 3-block of $S L_{2}(5)$ and the principal 3-block of $\mathfrak{A}_{5}$. Details are found in Example 2.2 below.

The paper is organised as follows. In Section 2 the above-cited examples are developed in detail. Section 3 is the technical heart of the paper since there Proposition 3.3 is shown: Given a self-dual, right-projective $H \times G$-bimodule $M$ and a self-dual $G$ module $V$, Proposition 3.3 gives an explicit isomorphism between the $H$-module $M \otimes_{G} V$ and its dual. The proposition applies as well for stable equivalences of Morita type. Except unicity, most of what follows is valid in this more general setting. Moreover, Theorem A is proven there. Section 4 then proves Theorem B and Section 5 proves Theorem C. 


\section{EXAMPLES}

In this section we show that Morita equivalence does not need to preserve self-duality of simple modules. In case it does, examples show that the geometric type of simple modules may vary via the correspondence.

In order to state the examples we need that blocks are determined by their Brauer trees up to Morita equivalence. Kupisch determined the structure of indecomposable modules of a Brauer tree algebra, and hence he implicitly determined their module category, i.e., the Morita equivalence class of a Brauer tree algebra. In modern terms Kupisch's result reads as follows.

Theorem 2.1. (Kupisch [7, 8]) Let $A_{1}$ and $A_{2}$ be two Brauer tree algebras. Then $A_{1}$ and $A_{2}$ are Morita equivalent if and only if their Brauer trees, including the exceptional vertex and its multiplicity, coincide.

In the following examples we shall use Brauer trees computed by Hiss and Lux in [5]. The geometric type of simple self-dual modules, i.e. symmetric or alternating, is taken from [1] or [6].

Example 2.2. Let $k$ be an algebraically closed field of characteristic 3 and let $G=S L_{2}(5)$ be the double cover of the alternating group $\mathfrak{A}_{5}$ of degree 5 . Then a non principal 3-block, say $B_{1}(G)$, of $G$ is a Brauer tree algebra corresponding to the Brauer tree

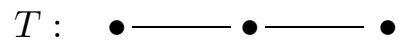

without an exceptional vertex. The ordinary characters of the algebra have dimensions 2, 4 and 2. Both of the simple modules in characteristic 3 of $B_{1}(G)$ admit an, up to scalar unique, $G$-invariant bilinear form. This form is alternating for both modules.

The principal 3-block of the alternating group $H=\mathfrak{A}_{5}$ is also a Brauer tree algebra with Brauer tree $T$. The ordinary characters of this algebra have dimensions 1,5 and 4 . In characteristic 3 both simple modules admit an, up to scalar unique, $\mathfrak{A}_{5}$-invariant bilinear form which is symmetric in both cases.

As a consequence, the type of bilinear forms is usually not preserved by Morita equivalence, even if self-duality is preserved on simple modules.

Example 2.3. Let $k$ be again an algebraically closed field but of characteristic 7 and let $G=3 M c L$ be the triple cover of a the McLaughlin simple group $M c L$.

Then, a non principal 7-block, say $B_{2}(3 M c L)$, of $G$ is a Brauer tree algebra corresponding to the Brauer tree

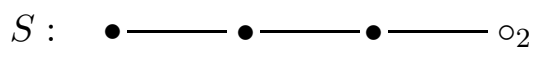

with an exceptional vertex $\mathrm{O}_{2}$ of multiplicity 2 . The ordinary characters of the algebra have dimensions 792, 4752, 6336 and 2376 read from the left in the order of the tree. None of the three simple modules in characteristic 7 is self-dual, hence none of them admit a $G$-invariant bilinear form.

The principal 7-block $B_{3}\left(M_{23}\right)$ of the Mathieu group $H=M_{23}$ is a Brauer tree algebra also with Brauer tree $S$. The ordinary characters of the algebra have dimensions 1, 1035, 2024 and 990 read from the left. The exceptional vertex corresponds to the two ordinary characters of degree 990. All simple modules in characteristic 7 admit an, up to scalar unique, $H$-invariant bilinear form which is symmetric in all three cases. 
As a consequence every Morita bimodule inducing a Morita equivalence between $B_{3}\left(M_{23}\right)$ and $B_{2}(3 M c L)$ has the property that each self-dual simple $B_{3}\left(M_{23}\right)$-module is sent to a simple $B_{2}(3 M c L)$-module which is not self-dual.

\section{Self-dual Morita Bimodules}

Let $H$ and $G$ be finite groups and let $k$ be a field. Furthermore, let $M$ be an $H \times G$ bimodule where $H$ acts on the left and $G$ on the right. Then $M^{*}=H_{o m}(M, k)$ becomes an $H \times G$-bimodule by setting

$$
(h f g) m=f\left(h^{-1} m g^{-1}\right)
$$

for $h \in H, g \in G, f \in M^{*}$ and $m \in M$. Indeed, the module structure follows by

$$
\begin{aligned}
\left(\left(h_{2} h_{1}\right) f\left(g_{1} g_{2}\right)\right) m & =f\left(\left(h_{2} h_{1}\right)^{-1} m\left(g_{1} g_{2}\right)^{-1}\right) \\
& =f\left(h_{1}^{-1} h_{2}^{-1} m g_{2}^{-1} g_{1}^{-1}\right) \\
& =\left(h_{1} f g_{1}\right)\left(h_{2}^{-1} m g_{2}^{-1}\right) \\
& =\left(h_{2}\left(h_{1} f g_{1}\right) g_{2}\right) m .
\end{aligned}
$$

Definition 3.1. Let $M$ be an $H \times G$-bimodule. A k-bilinear form $(\cdot, \cdot)$ on $M$ is called $H \times G$-invariant if

$$
(h m g, h n g)=(m, n)
$$

for all $h \in H$, all $g \in G$ and all $m, n \in M$.

The next lemma is well-known. For the readers convenience we give the proof.

Lemma 3.2. Let $M$ be an $H \times G$-bimodule. Then $M$ is isomorphic to $M^{*}$ as an $H \times G$ bimodule if and only if $M$ carries a non-degenerate $H \times G$-invariant bilinear form. More precisely, if $\varphi: M \rightarrow M^{*}$ is an isomorphism, then $\left(m, m^{\prime}\right):=\varphi(m)\left(m^{\prime}\right)$ is a non-degenerate $H \times G$-invariant bilinear form. If $(\cdot, \cdot)$ is a non-degenerate $H \times G$-invariant bilinear form, then the map $M \ni m \mapsto(\cdot, m) \in M^{*}$ is an isomorphism of $H \times G$-bimodules.

Proof. If $\varphi: M \rightarrow M^{*}$ denotes an $H \times G$-isomorphism we put

$$
(m, n):=\varphi(m) n
$$

for $m, n \in M$. Clearly, the form $(\cdot, \cdot)$ is $k$-linear, but it is also $H \times G$-invariant since

$$
\begin{aligned}
& (h m g, h n g)=\varphi(h m g)(h n g) \\
& =(h \varphi(m) g)(h n g) \quad(\text { since } \varphi \text { is } H \times G \text {-linear }) \\
& =\varphi(m)\left(h^{-1}(h n g) g^{-1}\right) \quad\left(\text { the module structure of } M^{*}\right) \\
& =\varphi(m) n \\
& =(m, n) \text {. }
\end{aligned}
$$

In addition $(\cdot, \cdot)$ is non-degenerate since $\varphi$ is an isomorphism.

Conversely, if $(\cdot, \cdot)$ is a non-degenerate $H \times G$-invariant bilinear form on $M$ then we put

$$
\varphi(m) n:=(m, n)
$$

for all $m, n \in M$. Note that $\varphi$ is a $k$-isomorphism since the bilinear form is non-degenerate. Furthermore, $\varphi$ is $H \times G$-linear since

$$
(h m g, n)=\varphi(h m g) n
$$

and

$$
(h m g, n)=\left(m, h^{-1} n g^{-1}\right)=\varphi(m)\left(h^{-1} n g^{-1}\right)=(h \varphi(m) g) n
$$

for all $h \in H, g \in G$ and $m, n \in M$. 
Proposition 3.3. Let $M$ be a self-dual $H \times G$-bimodule which is projective as a $G$-module. If $V$ is a self-dual $G$-left module then $M \otimes_{k G} V$ is a self-dual $H$-left module. More precisely, if $\beta: M \rightarrow M^{*}$ is an $H \times G$-linear isomorphism and $\alpha: V \rightarrow V^{*}$ is a $G$-linear isomorphism, then

$$
\begin{aligned}
M \otimes_{k G} V & \longrightarrow\left(M \otimes_{k G} V\right)^{*} \\
m \otimes v & \mapsto\left(m^{\prime} \otimes v^{\prime} \mapsto \sum_{g \in G} \beta\left(m g^{-1}\right)\left(m^{\prime}\right) \cdot \alpha(v)\left(g^{-1} v^{\prime}\right)\right)
\end{aligned}
$$

is an H-linear isomorphism.

Proof. Let $\beta: M \rightarrow M^{*}$ denote an $H \times G$-isomorphism and $\alpha: V \rightarrow V^{*}$ a $G$-isomorphism. Therefore, since $\alpha$ and $\beta$ are $k G$-linear the map $\gamma:=\beta \otimes_{k G} \alpha: M \otimes_{k G} V \rightarrow M^{*} \otimes_{k G} V^{*}$ is a well-defined $k$-vector space isomorphism satisfying $\left(\beta \otimes_{k G} \alpha\right)\left(m \otimes_{k G} v\right)=\beta(m) \otimes_{k G} \alpha(v)$ for all $m \in M$ and $v \in V$ (cf. e.g. [2, Theorem 12.10]).

Now, let $m \in M, v \in V$ and $h \in H$. Since

$$
\begin{aligned}
\gamma\left(h\left(m \otimes_{k G} v\right)\right) & =\gamma\left(h m \otimes_{k G} v\right) \\
& =\beta(h m) \otimes_{k G} \alpha(v) \\
& =h \beta(m) \otimes_{k G} \alpha(v) \quad \text { (since } \beta \text { is } H \text {-linear) } \\
& =h \gamma\left(m \otimes_{k G} v\right) .
\end{aligned}
$$

the map $\gamma$ is $H$-linear.

Finally, we claim that

$$
M^{*} \otimes_{k G} V^{*} \cong\left(M \otimes_{k G} V\right)^{*}
$$

as $H$-left modules. We prove this via a sequence of isomorphisms.

Observe that $V^{*}$ is not only a $G$-left but in a natural way also a $G$-right module via the definition

$$
(f g) v=f(g v)
$$

for $f \in V^{*}, g \in G$ and $v \in V$.

Considering $V^{*}$ as $G$-right module $\operatorname{Hom}_{k G}\left(M, V^{*}\right)$ makes sense. With this observation we have

Claim (i) $\operatorname{Hom}_{k G}\left(M, V^{*}\right) \cong \operatorname{Hom}_{k}\left(M \otimes_{k G} V, k\right)=\left(M \otimes_{k G} V\right)^{*}$ as $H$-left modules.

The isomorphism is given by sending $\alpha \in \operatorname{Hom}_{k G}\left(M, V^{*}\right)$ to the map $\Phi(\alpha): M \otimes_{k G} V \rightarrow k$ with $\Phi(\alpha)\left(m \otimes_{k G} v\right)=\alpha(m) v$ where $m \in M$ and $v \in V$. Note, that $\Phi$ is well-defined since $\alpha$ is $G$-linear, for

$$
\Phi(\alpha)\left(m g \otimes_{k G} v\right)=\alpha(m g)(v)=(\alpha(m) g)(v)=\alpha(m)(g v)=\Phi(\alpha)\left(m \otimes_{k G} g v\right) .
$$

Furthermore, $\Phi$ is $H$-linear since

$$
\Phi(h \alpha)\left(m \otimes_{k G} v\right)=(h \alpha)(m) v=\alpha\left(h^{-1} m\right) v=\Phi(\alpha)\left(h^{-1} m \otimes_{k G} v\right)=(h \Phi(\alpha))\left(m \otimes_{k G} v\right) .
$$

Finally, $\alpha \rightarrow \Phi(\alpha)$ is obviously a monomorphism. In order to show that $\Phi$ is an isomorphism we define a $k$-linear map

$$
\Psi: \operatorname{Hom}_{k}\left(M \otimes_{k G} V, k\right) \rightarrow H o m_{k G}\left(M, V^{*}\right)
$$

by putting $(\Psi(\varphi)(m))(v):=\varphi(m \otimes v)$ for $\varphi \in H_{o m}\left(M \otimes_{k G} V, k\right), m \in M$ and $v \in V$. Since $\varphi$ is defined over $M \otimes_{k G} V$ we have

$$
(\Psi(\varphi)(m g)) v=\varphi\left(m g \otimes_{k G} v\right)=\varphi\left(m \otimes_{k G} g v\right)=(\Psi(\varphi)(m))(g v)=(\Psi(\varphi)(m) g) v
$$


for $m \in M, g \in G$ and $v \in V$. Thus $\Psi(\varphi) \in \operatorname{Hom}_{k G}\left(M, V^{*}\right)$. Moreover,

$$
((\Phi \circ \Psi)(\varphi))(m \otimes-)=\Phi(m \mapsto \varphi(m \otimes-))=\varphi(m \otimes-)
$$

for $m \in M$. Thus $\Phi$ is surjective, hence an isomorphism.

Claim (ii) $\operatorname{Hom}_{k G}(M, k G) \otimes_{k G} V^{*} \cong \operatorname{Hom}_{k G}\left(M, V^{*}\right)$ as $H$-left modules:

The isomorphism is given by

$$
\alpha \otimes_{k G} f \mapsto \widehat{\alpha \otimes_{k G} f}
$$

for $\alpha \in \operatorname{Hom}_{k G}(M, k G)$ and $f \in V^{*}$ where

$$
\left(\left(\widehat{\alpha \otimes_{k G}} f\right)(m)\right)(v)=(f \alpha(m))(v)=f(\alpha(m) v)
$$

for $m \in M$ and $v \in V$. Here $V^{*}$ is considered as a $G$-right module by the natural action as defined above. Furthermore, the space $\operatorname{Hom}_{k G}(M, k G)$ is an $H \times G$-bimodule via the action

$$
(h f g)(m):=g^{-1} f\left(h^{-1} m\right)
$$

for $m \in M, g \in G, h \in H$ and $f \in \operatorname{Hom}_{k G}(M, k G)$.

First we show that the map $>$ is well-defined.

Indeed, for $f \in V^{*}, g \in G, \alpha \in \operatorname{Hom}_{k G}(M, k G), v \in V$ and $m \in M$ one have

$$
\begin{array}{rlr}
\left(\left((\alpha g) \widehat{\otimes_{k} G} f\right)(m)\right)(v) & =(f((\alpha g)(m)))(v) & \text { (definition of }- \text { ) } \\
& =f(((\alpha g)(m)) v) & \left.\quad \text { (right module structure of } V^{*}\right) \\
& =f\left(\left(g^{-1}(\alpha(m))\right) v\right) & \text { (right module structure of } \left.\operatorname{Hom}_{k G}(M, k G)\right) \\
& =f\left(g^{-1}(\alpha(m) v)\right) & \left.\quad \text { (associativity of the } k G \text {-action on } V^{*}\right) \\
& =(g f)(\alpha(m) v) & \left.\quad \text { (left module structure of } V^{*}\right) \\
& =\left(\left(\alpha \widehat{\otimes_{k G}(g f)}\right)(m)\right)(v) \quad \text { (definition of }- \text { ) }
\end{array}
$$

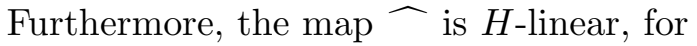

$$
\left(h \widehat{\alpha \otimes_{k G}} f\right)(m)=f(h \alpha)(m)=f \alpha\left(h^{-1} m\right)=\left(\widehat{\alpha \otimes_{k G}} f\right)\left(h^{-1} m\right)=\left(h\left(\widehat{\alpha \otimes_{k G}} f\right)\right)(m) .
$$

Thus it remains to prove that $\widehat{-}$ is a $k$-isomorphism. First we consider the case $M=k G$. Let $\alpha \in \operatorname{Hom}_{k G}\left(k G, V^{*}\right)$, hence

$$
\alpha\left(\sum_{g \in G} a_{g} g\right)=\sum_{g \in G} a_{g} \alpha(g)=\sum_{g \in G} a_{g} \alpha(1) g=f \sum_{g \in G} a_{g} g
$$

where $\alpha(1)=f \in V^{*}$ and $a_{g} \in k$ for $g \in G$. If $i d$ denotes the identity in $\operatorname{Hom}_{k G}(k G, k G)$ then

$$
\left(i \widehat{\otimes_{k G}} f\right)\left(\sum_{g \in G} k_{g} g\right)=f \sum_{g \in G} k_{g} g=\alpha\left(\sum_{g \in G} k_{g} g\right),
$$

hence $i \widehat{\otimes_{k G}} f=\alpha$. Thus the above map is an epimorphism, and therefore an isomorphism since the dimensions of the spaces in (ii) are obviously equal for $M=k G$. Since any free $G$-module is a direct sum of modules isomorphic $k G$ the map - is also an isomorphism if $M$ is a free module. Since a projective module is a direct summand of a free module and since the isomorphisms are all compatible with taking direct summands the proof of (ii) is complete.

Claim (iii) $\operatorname{Hom}_{k}(M, k) \cong \operatorname{Hom}_{k G}(M, k G)$ as $H \times G$-bimodules:

Abstractly this follows by Frobenius reciprocity [9, Chapter VI, formula (8.7)] and the fact that $k G$ is a symmetric algebra. However, we need the isomorphism explicit. Let $(\cdot, \cdot)$ be 
the symmetrising bilinear form making $k G$ into a symmetric algebra. For all $x, y \in k G$ the value of $(x, y)$ is the coefficient of 1 in $x y \in k G$ (cf. e.g. [2, Theorem 62.1]). Then the map

$$
\begin{aligned}
\operatorname{Hom}_{k G}(M, k G) & \stackrel{\rho_{M}}{\longrightarrow} \operatorname{Hom}_{k}(M, k) \\
f & \mapsto(m \mapsto(f(m), 1))
\end{aligned}
$$

is a $k$-linear. If $\rho_{M}(f)=0$ we get

$$
0=(f(m x), 1)=(f(m) x, 1)=(f(m), x)
$$

for all $m \in M$ and all $x \in k G$. Since $(\cdot, \cdot)$ is non degenerate $f(m)=0$ for all $m \in M$ and hence $f=0$. Therefore $\rho_{M}$ is injective.

Moreover, $\rho_{M}$ is a morphism of $H \times G$-bimodules. Indeed, for $g \in G$ and $h \in H$, for $f \in \operatorname{Hom}_{k G}(M, k G)$ and $m \in M$, we have

$$
\begin{aligned}
& \left(\rho_{M}(h f g)\right)(m)=((h f g)(m), 1) \quad\left(\text { definition of } \rho_{M}\right) \\
& =\left(g^{-1} f\left(h^{-1} m\right), 1\right) \quad\left(H \times G \text {-bimodule structure of } \operatorname{Hom}_{k G}(M, k G)\right) \\
& =\left(1, g^{-1} f\left(h^{-1} m\right)\right) \quad(\text { since }(\cdot, \cdot) \text { is symmetric) } \\
& =\left(g^{-1}, f\left(h^{-1} m\right)\right) \quad(\text { since }(\cdot, \cdot) \text { is associative }) \\
& =\left(f\left(h^{-1} m\right), g^{-1}\right) \quad(\text { since }(\cdot, \cdot) \text { is symmetric) } \\
& =\left(f\left(h^{-1} m\right) g^{-1}, 1\right) \quad(\text { since }(\cdot, \cdot) \text { is associative }) \\
& =\left(f\left(h^{-1} m g^{-1}\right), 1\right) \quad(f \text { is } k G \text {-linear }) \\
& =\rho_{M}(f)\left(h^{-1} m g^{-1}\right) \quad\left(\text { definition of } \rho_{M}\right) \\
& =\left(h\left(\rho_{M}(f)\right) g\right)(m) \quad\left(H \times G \text {-bimodule structure of } \operatorname{Hom}_{k}(M, k)\right)
\end{aligned}
$$

and so $\rho_{M}(h f g)=h\left(\rho_{M}(f)\right) g$ which shows that $\rho_{M}$ is $H \times G$-linear.

The inverse isomorphism is given by sending $\varphi \in \operatorname{Hom}_{k}(M, k)$ to $\Lambda_{M}(\varphi) \in \operatorname{Hom}_{k G}(M, k G)$ defined by

$$
\Lambda_{M}(\varphi)(m)=\sum_{x \in G} \varphi\left(m x^{-1}\right) x
$$

for $m \in M$ : First note, that $\Lambda_{M}(\varphi)$ is $G$-linear since it is the trace under the $G$-action. Furthermore,

$$
\begin{aligned}
\left(\left(\rho_{M} \circ \Lambda_{M}\right)(\varphi)\right)(m) & =\left(\sum_{x \in G} \varphi\left(m x^{-1}\right) x, 1\right) \\
& =\sum_{x \in G} \varphi\left(m x^{-1}\right)(x, 1) \\
& =\sum_{x \in G} \varphi\left(m x^{-1}\right) \delta_{x, 1} \\
& =\varphi(m)
\end{aligned}
$$

for $m \in M$ and $\varphi \in \operatorname{Hom}_{k}(M, k)$ where $\delta$ denotes the Kronecker symbol. Thus, $\rho_{M}$ is surjective and $\rho_{M}^{-1}=\Lambda_{M}$ is an isomorphism of $H \times G$-bimodules. 
Since $\Lambda_{M}$ is $H \times G$-linear, $\Lambda_{M} \otimes_{k G} i d_{V^{*}}$ is well defined (cf. e.g. [2, Theorem 12.10]). Summarising (i), (ii) and (iii) we get explicit isomorphisms

$$
\begin{array}{rll}
M \otimes_{k G} V & \stackrel{\beta \otimes \alpha}{\longrightarrow} & M^{*} \otimes_{k G} V^{*} \\
\stackrel{\Lambda_{M} \otimes i d_{V^{*}}}{\longrightarrow} & \operatorname{Hom}_{k G}(M, k G) \otimes_{k G} V^{*} \\
& \longrightarrow & \operatorname{Hom}_{k G}\left(M, V^{*}\right) \\
& \stackrel{\Phi}{\longrightarrow} \operatorname{Hom}_{k}\left(M \otimes_{k G} V, k\right)=\left(M \otimes_{k G} V\right)^{*}
\end{array}
$$

as $H$-left modules.

We need to verify the formula given in the Proposition.

$$
\begin{aligned}
m \otimes v & \stackrel{\beta \otimes \alpha}{\mapsto}(m) \otimes_{k G} \alpha(v) \\
\stackrel{\Lambda_{M} \otimes i d}{\mapsto} & \sum_{g \in G} \beta\left(m g^{-1}\right) g \otimes_{k G} \alpha(v) \\
& =\sum_{g \in G} \beta\left(m g^{-1}\right) \otimes_{k G} g \alpha(v) \\
& \mapsto \quad m^{\prime} \mapsto\left(v^{\prime} \mapsto\left(\sum_{g \in G} g \alpha(v)\left(\beta\left(m g^{-1}\right)\left(m^{\prime}\right) v^{\prime}\right)\right)\right) \\
& =m^{\prime} \mapsto\left(v^{\prime} \mapsto\left(\sum_{g \in G} \beta\left(m g^{-1}\right)\left(m^{\prime}\right) \cdot g \alpha(v)\left(v^{\prime}\right)\right)\right) \\
& \left(\left(m^{\prime} \otimes v^{\prime}\right) \mapsto\left(\sum_{g \in G} \beta\left(m g^{-1}\right)\left(m^{\prime}\right) \cdot \alpha(v)\left(g^{-1} v^{\prime}\right)\right)\right)
\end{aligned}
$$

This complets the proof.

The following is the translation of the isomorphism given in Proposition 3.3 in terms of bilinear forms.

Corollary 3.4. Let $M$ be an $H \times G$-bimodule which is projective as a $G$-module. Suppose that $M$ carries a non-degenerate $H \times G$-invariant non-degenerate bilinear form $b$. Let $V$ be a $G$-left module with a non-degenerate $G$-invariant bilinear form $B$. Then, for $m, m^{\prime} \in M$ and $v, v^{\prime} \in V$,

$$
\tilde{B}\left(m \otimes_{k G} v, m^{\prime} \otimes_{k G} v^{\prime}\right)=\sum_{g \in G} b\left(m g^{-1}, m^{\prime}\right) B\left(v, g^{-1} v^{\prime}\right)
$$

defines a non-degenerate $H$-invariant bilinear form on $M \otimes_{k G} V$.

Proof. By Lemma 3.2, the bilinear form $b$ is associated to an $H \times G$-isomorphism $\beta: M \rightarrow M^{*}$ via

$$
b\left(m, m^{\prime}\right)=\beta(m) m^{\prime}
$$

for $m, m^{\prime} \in M$. Similarly, $B$ is associated to a $G$-isomorphism $\alpha: V \rightarrow V^{*}$ via

$$
B\left(v, v^{\prime}\right)=\alpha(v) v^{\prime}
$$

for $v, v^{\prime} \in V$. Proposition 3.3 shows that an isomorphism

$$
\Psi: M \otimes_{k G} V \longrightarrow\left(M \otimes_{k G} V\right)^{*}
$$


is given by

$$
\begin{aligned}
(\Psi(m \otimes v))\left(m^{\prime} \otimes v^{\prime}\right) & =\sum_{g \in G} \beta\left(m g^{-1}\right)\left(m^{\prime}\right) \cdot \alpha(v)\left(g^{-1} v^{\prime}\right) \\
& =\sum_{g \in G} b\left(m g^{-1}, m^{\prime}\right) B\left(v, g^{-1} v^{\prime}\right) .
\end{aligned}
$$

Thus, by Lemma 3.2, the bilinear form $\tilde{B}$ defined on $M \otimes_{k G} V$ by

$$
\tilde{B}\left(m \otimes_{k G} v, m^{\prime} \otimes_{k G} v^{\prime}\right)=\sum_{g \in G} b\left(m g^{-1}, m^{\prime}\right) B\left(v, g^{-1} v^{\prime}\right)
$$

is non-degenerate and $H$-invariant.

Proof of Theorem A. By Proposition 3.3 , the functor $M \otimes_{k G}-$ maps self-dual $G$-modules to self-dual $H$-modules.

On the other hand, $\operatorname{Hom}_{k H}(M, k H)$ becomes a $G \times H$-bimodule via

$$
(g \alpha h)(m)=\alpha(m g) h
$$

for all $g \in G, h \in H$ and $\alpha \in H_{o m}(M, k H)$. Then $\operatorname{Hom}_{k H}(M, k H)$ is the inverse Morita bimodule to $M$ and hence $\operatorname{Hom}_{k H}(M, k H)$ defines the inverse functor from the category of $B_{H}$-modules onto the category of $B_{G}$-modules. In order to prove the only if part it is, by Proposition 3.3, enough to show that the $G \times H$-bimodule $\operatorname{Hom}_{k H}(M, k H)$ is self-dual as well.

First note that

$$
\operatorname{Hom}_{k H}(M, k H) \cong \operatorname{Hom}_{k}(M, k)={ }_{G}\left(M^{*}\right)_{H}
$$

as $G \times H$-bimodules (by Claim (iii) in the proof of Proposition 3.3 , changing left- and right structures there). If we define a $G \times H$-structure on $M$ by setting

$$
g \circ m \circ h=h^{-1} m g^{-1}
$$

then the inverse of the given isomorphism ${ }_{H} M_{G} \longrightarrow{ }_{H} M_{G}^{*}$ defines an isomorphism from ${ }_{G}\left(M^{*}\right)_{H}$ to $M \cong\left({ }_{G}\left(M^{*}\right)_{H}\right)^{*}$ and we are done.

Example 2.3 shows that not all Morita bimodules do admit a non-degenerate invariant bilinear form.

Remark 3.5. Given two Morita equivalences between two blocks $B_{G}$ and $B_{H}$ of group algebras $k G$ and $k H$, the composition of the one with the inverse of the other gives a Morita self-equivalence of the block $B_{G}$. Now, the isomorphism classes of Morita self-equivalences of $B_{G}$ form the Picard group $\operatorname{Pic}\left(B_{G}\right)$ which is isomorphic to the outer automorphism group $\operatorname{Out}\left(B_{G}\right):=\operatorname{Aut}_{k-\operatorname{alg}}\left(B_{G}\right) / \operatorname{Inn}\left(B_{G}\right)$ where as usual $\operatorname{Inn}\left(B_{G}\right)$ denotes the inner automorphisms of $B_{G}$. Each $\alpha \in A u t_{k-\operatorname{alg}}\left(B_{G}\right)$ induces an equivalence by sending a module $V$ to ${ }^{\alpha} V$, which is defined to be $V$ as a $k$-vector space, but where the action of $B_{G}$ on ${ }^{\alpha} V$ is given by

$$
a \bullet v=\alpha(a) v
$$

for $a \in B_{G}$ and $v \in V$. Since $\left({ }^{\alpha} V\right)^{*} \cong{ }^{\alpha}\left(V^{*}\right)$ the automorphism $\alpha$ preserves the property of self-duality on modules. Since $B_{H} \simeq \operatorname{End}_{B_{G}}(M)$, whatever isomorphism is taken to identify $B_{H}$ with $\operatorname{End}_{B_{G}}(M)$ the question whether a given module $M \otimes_{k G} V$ carries a non degenerate $H$-invariant bilinear form is independent of this choice.

There are Morita equivalences which are induced by self-dual bimodules.

Example 3.6. Let $G$ be a finite $p$-solvable group and let $N=O_{p^{\prime}}(G)$. We put $H=G / N$ and

$$
e=\frac{1}{\left|O_{p^{\prime}}(G)\right|} \sum_{g \in O_{p^{\prime}}(G)} g .
$$


Clearly, $e$ is the block idempotent of the principal block $B_{0}$ of $k G$ and $e k G \cong k G / N=k H$ as $H \times G$-bimodules where $N$ obviously acts trivially on both sides. The bimodule ${ }_{H} M_{G}=e k G$ is a Morita bimodule which induces an equivalence between the principal block of $k G$ and the group algebra $k H$. In fact

$$
\operatorname{End}_{k G}(e k G) \cong e k G e \cong k G / N=k H,
$$

and obviously $e k G$ is a progenerator of $e k G=B_{0}$. Moreover, ekG $\cong(e k G)^{*}$ as $H \times G$ bimodules since, by a well-known result on duality,

$$
(e k G)^{*} \cong \bar{e} k G=e k G
$$

where ${ }^{-}$denotes the anti-automorphism of $k G$ defined by $g \rightarrow \bar{g}=g^{-1}$. Thus the equivalence preserves self-duality, by Proposition 3.3. Clearly, this fact is obvious since $N$ acts trivially on all modules in $B_{0}$.

\section{Morita EQUivalence AND FORMS In OdD CHARACTERISTIC}

Throughout this section let $k$ be a field of odd characteristic (which includes the characteristic 0 case as well). A $G$-module $V$ is called of symmetric resp. antisymmetric type if $V$ carries a non-degenerate $G$-invariant symmetric resp. antisymmetric bilinear form.

Proposition 4.1. Let $M$ be an $H \times G$-bimodule inducing a Morita equivalence between a $k G$-block $B_{G}$ and a $k H$-block $B_{H}$. Suppose that $M$ is of symmetric type. Let $V$ be a self-dual $G$-left module. If $V$ is of symmetric resp. antisymmetric type then $M \otimes_{k G} V$ is of symmetric resp. antisymmetric type.

Proof. Suppose $b$ is a symmetric bilinear form and the bilinear form $B$ is symmetric resp. antisymmetric if $V$ is of symmetric resp. antisymmetric type. With the notation of Corollary [3.4, we have

$$
\begin{aligned}
\tilde{B}\left(m \otimes_{k G} v, m^{\prime} \otimes_{k G} v^{\prime}\right) & =\sum_{g \in G} b\left(m g^{-1}, m^{\prime}\right) B\left(v, g^{-1} v^{\prime}\right) \\
& =\sum_{g \in G} b\left(m, m^{\prime} g\right) B\left(g v, v^{\prime}\right) \quad \text { (since } b \text { and } B \text { are } G \text {-invariant) } \\
& \left.= \pm \sum_{g \in G} b\left(m^{\prime} g, m\right) B\left(v^{\prime}, g v\right) \quad \text { (by symmetry of } b \text { and } B\right) \\
& = \pm \tilde{B}\left(m^{\prime} \otimes_{k G} v^{\prime}, m \otimes_{k G} v\right),
\end{aligned}
$$

where the + sign appears for symmetric and the - sign for antisymmetric type modules $V$.

Proof of Theorem B. This is an immediate consequence of Proposition 4.1,

Example 4.2. We consider again Example 3.6. Note that the Morita module $e k G$ is of symmetric type. As a form one can take the standard non-degenerate $G$-invariant symmetric form on $k G$ defined by

$$
b\left(g, g^{\prime}\right)=\delta_{g, g^{\prime}}
$$

for $g, g^{\prime} \in G$ restricted to $e k G$. The restriction is indeed non-degenerate as one can check easily. Thus Proposition 4.1 applies.

Now let $V$ be a simple self-dual $k G$-module. Thus $V$ carries a $G$-invariant non-degenerate bilinear form. If $k$ is algebraically closed then - up to scalars - there is exactly one nondegenerate $G$-invariant form which is symmetric or antisymmetric since the characteristic is odd ([4, Chap. VII, 8.12). Thus, for $k$ algebraically closed, a simple self-dual $G$-module is either of symmetric or antisymmetric type but not of both. In particular, the Morita module $M=e k G$ in Example 4.2 is never of antisymmetric type since otherwise, by Proposition 4.1. there are simple $H$-modules which are of symmetric and antisymmetric type. Indeed it is well-known, that a group algebra $k H \cong e k G$ in odd characteristic never has a non-degenerate $H$-invariant antisymmetric form. 
Remark 4.3. Let $k$ be an algebraically closed field of odd characteristic. By definition, a Morita bimodule $M={ }_{H} M_{G}$ is a projective $k G$-right and a projective $k H$-left module. If $M$ carries a non-degenerate $H \times G$-invariant symmetric form then a projective indecomposable $k G$-resp. $k H$-module of antisymmetric type occurs with even multiplicity in $M$. Note that, by ([12], Proposition 2.2), an indecomposable projective module is of antisymmetric type if and only if its head is of antisymmetric type.

\section{Morita EQUiVAlENCE AND FORMS IN EVEN CHARACTERISTIC}

In characteristic 2 the geometry of self-dual modules turns out to be more subtle. In this case an alternating form is symmetric. By Fong's Lemma (44, Chap. VII, Theorem 8.13), a simple non-trivial self-dual $k G$-module $V$ always carries a non-degenerate $G$-invariant symplectic form, but it may happen that $V$ even has a non-degenerate $G$-invariant quadratic form. This happens if $H^{1}(G, V)=0$ ([11], Proposition 2.4) and a connecting homomorphism to the first cohomology group carries the essential information what really happens. We call an arbitrary module $V \cong V^{*}$ of quadratic type if it carries a non-degenerate $G$-invariant quadratic form, say $Q$. The corresponding $G$-invariant non-degenerate symplectic form $B$ defined by

$$
B\left(v, v^{\prime}\right)=Q\left(v+v^{\prime}\right)-Q(v)-Q\left(v^{\prime}\right)
$$

for $v, v^{\prime} \in V$ is called the Polarisation of $Q$. Note that the quadratic form $Q$ is non-degenerate if

$$
\operatorname{rad} Q:=\{v \mid v \in \operatorname{rad} B, Q(v)=0\}=0 .
$$

Throughout the following $k$ is assumed to be a perfect field of characteristic 2. The next Lemma can be found as Proposition 2.2 in [3].

Lemma 5.1. Let $P$ be a projective $k G$-module not containing the projective cover of the trivial module. Then each $G$-invariant symmetric form on $P$ is the Polarisation of a $G$ invariant quadratic form on $P$.

We are now ready to prove Theorem $\mathrm{C}$.

Proof of Theorem $C$. Let $P$ be a projective $k G$-module of quadratic type. Then $P$ is an orthogonal sum of orthogonal indecomposable modules. By ([12, Lemma 3.6), an orthogonal indecomposable module is either indecomposable or of the form $S \oplus S^{*}$ where $S$ is indecomposable. Since

$$
\left(M \otimes_{k G}\left(S \oplus S^{*}\right)\right)^{*} \cong M^{*} \otimes_{k G}\left(S \oplus S^{*}\right)^{*} \cong M \otimes_{k G}\left(S \oplus S^{*}\right)
$$

by Claim (i), (ii) and (iii) in the proof of Proposition 3.3 we need to prove the Theorem only for orthogonal indecomposable projective modules.

So let $P$ be an orthogonal indecomposable projective $k G$-module in $B_{G}$ of quadratic type. Thus $P$ carries a non-degenerate $G$-invariant symplectic form $B$ which is also symmetric. According to Corollary 3.4 the form $\tilde{B}$ defined by

$$
\tilde{B}\left(m \otimes_{k G} p, m^{\prime} \otimes_{k G} p^{\prime}\right)=\sum_{g \in G} b\left(m g^{-1}, m^{\prime}\right) B\left(p, g^{-1} p^{\prime}\right)
$$

for $m, m^{\prime} \in M$ and $p, p^{\prime} \in P$ is a non-degenerate $H$-invariant bilinear form on $M \otimes_{k G} P$. Since $b$ and $B$ are symmetric the form $\tilde{B}$ is symmetric as well. Thus, if the projective module $M \otimes_{k G} P$ does not contain the projective cover of the trivial $k H$-module, say $P_{H}(1)$, then $M \otimes_{k G} P$ is of quadratic type, by Lemma 5.1. In case it contains $P_{H}(1)$ it is either $P_{H}(1)$ or a direct sum of two copies of $P_{H}(1)$. But, by ([12], Remark $\left.3.5(\mathrm{~b})\right), P_{H}(1)$ and hence any finite direct sum of it is always of quadratic type if 2||$H \mid$. In case $2 \nmid|H|$ the trivial module $k$ is projective and obviously of quadratic type. This completes the proof. 
At the moment we are not able to prove an analogue of Theorem $\mathrm{C}$ for simple self-dual modules with the methods we have developed so far.

\section{REFERENCES}

[1] J.H. Conway, R.T. Curtis, S.P. Norton, R.A. Parker and R.A. Wilson Atlas of Finite Groups, Clarendon Press, Oxford 1985.

[2] Charles W. Curtis and Irving Reiner, Representation theory of finite groups and associative ALGEBRAS, J. Wiley and Sons New-York 1966.

[3] Roderick Gow and Wolfgang Willems, Quadratic geometries, projective modules, and idempotents, Journal of Algebra 160 (1993), 257-272.

[4] Bertram Huppert and Norman Blackburn, Finite Groups II, Springer Berlin 1982.

[5] Gerhard Hiss and Klaus Lux, Brauer trees of sporadic Groups, Oxford Science Publishers 1989.

[6] Christoph Jansen, Klaus Lux, Richard Parker and Robert Wilson, An Atlas of Brauer Characters, Clarendon Press, Oxford 1995.

[7] Herbert Kupisch, Projektive Moduln endlicher Gruppen mit zyklischer p-Sylow-Gruppe, Journal of Algebra 10 (1968) 1-7.

[8] Herbert Kupisch, Unzerlegbare Moduln endlicher Gruppen mit zyklischer p-Sylow-Gruppe, Mathematische Zeitschrift 108 (1969) 77-104.

[9] Saunders MacLane, Homology, Springer Verlag Berlin 1994.

[10] Irving Reiner, Maximal Orders, Academic press 1975.

[11] Peter Sin and Wolfgang Willems, G-invariant quadratic forms, Journal für die Reine und Angewandte Mathematik 420 (1991), 45-59.

[12] Wolfgang Willems, Duality and forms in representation theory, in Progress in Mathematics Vol. 95, Birkhäuser (1991), 509-520.

WOLFGANG WiLlems, Institut für Algebra und Geometrie, FAKULTÄT FÜr MATHEMATIK, OTTO-VON-GUERICKE-UNIVERSITÄT, 39016 Magdeburg,

GERMANY

E-mail address: wolfgang.willems@ovgu.de

AleXANDER ZimmermanN

Université DE PiCARDie,

FACUlté de Mathématiques et LAMFA (UMR 6140 DU CNRS),

33 RUE ST LEU,

F-80039 Amiens Cedex 1,

FRANCE

E-mail address: alexander.zimmermann@u-picardie.fr 